\title{
Prediction and Potential Preventions for the Development of Posttraumatic Osteoarthritis after the Terrible Triad Injury: A Multicenter Risk Factors Study
}

\author{
Jingjing Li, ${ }^{1}$ Di Lu, ${ }^{2}$ Wenxiao Lin, ${ }^{1}$ Qinglong $\mathrm{Li}^{3}{ }^{3}$ Jing Hu, ${ }^{4}$ Ding Xu $\mathbb{D},{ }^{5}$ Youming Zhao, ${ }^{2}$ \\ and Weijun Guo $\mathbb{D}^{2}$ \\ ${ }^{1}$ Department of Radiology, The Second Affiliated Hospital and Yuying Children's Hospital of Wenzhou Medical University, \\ Wenzhou, China \\ ${ }^{2}$ Department of Orthopedics, The Second Affiliated Hospital and Yuying Children's Hospital of Wenzhou Medical University, \\ Wenzhou, China \\ ${ }^{3}$ Department of Orthopedics, Sichuan Orthopedics Hospital, Sichuan, China \\ ${ }^{4}$ Department of Orthopedics, Wenzhou People's Hospital, Wenzhou, China \\ ${ }^{5}$ Department of Orthopedics, Shangyu People's Hospital, Shaoxing, China
}

Correspondence should be addressed to Weijun Guo; wjguo2015@sina.com

Received 25 October 2019; Revised 12 May 2020; Accepted 8 June 2020; Published 22 June 2020

Academic Editor: Gang Liu

Copyright (c) 2020 Jingjing Li et al. This is an open access article distributed under the Creative Commons Attribution License, which permits unrestricted use, distribution, and reproduction in any medium, provided the original work is properly cited.

Objective. A multicenter study to evaluate risk factors for the development of moderate or severe posttraumatic osteoarthritis (PTOA) and to find potential preventions. Methods. We conducted a retrospective multicenter study including the terrible triad injury (TTI) patients with surgical treatment from January 2007 to November 2014. Demographics, injury information, and treatment history were obtained retrospectively. According to the Broberg and Morrey criterion, 198 included patients were sorted into two groups: the mild or no PTOA and moderate or severe PTOA. Uni- and multivariate logistic regression analyses were used to identify risk factors for moderate or severe PTOA. Results. Moderate or severe PTOA was present in 64 patients (32.3\%). Significant risk factors were Mason III radial head fracture (OR 4.049, 95\% CI 1.877-8.736, $p<0.001$ ), medial collateral ligament injury (OR 5.120, 95\% CI 1.261-20.790, $p=0.022$ ), and heavy use of elbow (OR 2.333, 95\% CI 1.060-5.136, $p=0.035$ ). Besides, patients suffered subluxation $(p=0.007)$ and those with more risk factors had a higher risk to develop moderate or severe PTOA. Conclusions. Moderate or severe PTOA was common after the TTI. Patients need to be counseled about avoiding heavy use of the elbow, especially for those with Mason III radial head fractures. Surgeons should be aware of the recurrent instability of the elbow.

\section{Introduction}

Elbow dislocation associated with both radial head and coronoid fractures earned its eponym "terrible triad injury" (TTI) for decades because of the poor prognosis [1]. With the improvement of knowledge on elbow biomechanics and pathoanatomy, the current standard strategy appeared to have yielded more favorable outcomes [2, 3]. However, challenges persist. Complications like joint stiffness, posttraumatic osteoarthritis (PTOA), heterotopic ossification, and ulnar nerve symptoms continue to affect the prognosis of the TTI $[4,5]$.

PTOA is difficult to manage, especially in the highdemand or active population. It presents a challenge for both patients and orthopedic surgeons. The correlation between radiographic evidence of PTOA and symptoms is limited, especially in mild ones $[9,10]$. When PTOA has aggravated, several treatments would be considered for moderate or severe PTOA. Unfortunately, cartilage damage during these stages cannot be reversed [6]. Pharmacologic therapies 
(including anti-inflammatory agents, joint injection with corticosteroid or hyaluronic acid) offered only relief of pain and inflammation instead of any long term benefit [7]. Arthroplasty is recommended only for the older and lowdemand patients [8], whereas TTI is most common in midage patients who are the main labor force and difficult to comply with long-term activity restrictions. Increased demands placed throughout their lifetime make arthroplasty only a remedy option [9]. Hence, it is important to understand the risk factors for the development of moderate or severe PTOA to provide a theoretical basis for early prevention, while delaying elbow arthroplasty to the extent possible. But little is known.

Therefore, we designed a multicenter retrospective study of TTI patients after surgical treatment. We used uni- and multivariate logistic regression analyses to identify risk factors for the development of moderate or severe PTOA and to find potential preventions.

\section{Material and Methods}

2.1. Study Design and Patients. This study was approved by the Ethics Committee of our hospitals. We conducted a retrospective cohort study including consecutive patients operated upon at our four hospitals from January 2007 to November 2014 for the TTI of the elbow treated by ORIF. Inclusion criteria were (1) TTI patients with surgical treatment, (2) an age of 18 years or older, and (3) medical information with elbow radiograph of more than 3 years. Patients with pathological fractures, prior elbow trauma or osteoarthritis, or incomplete medical information were excluded, as well as those treated with arthroplasty or resection for radial head fractures.

Operations were performed by different surgeons based on the standard strategy [10]. In brief, coronoid fractures were treated with cannulated screws or miniscrews. Otherwise, the anterior capsular repair was performed for ReganMorrey type I and some type II fractures. Then, radial head fractures of Mason II and III were treated with cannulated screws or miniscrews, and small fragments were fixed by $\mathrm{K}$ wires. Plates were used for radial neck fractures. Mason I fractures were treated conservatively. All lateral collateral ligaments were repaired as well as the extensor origin and the posterolateral capsule. Afterward, elbow stability was evaluated. Medial collateral ligament (MCL) repair or hinged external fixator was performed according to the extent of valgus stability.

\subsection{Data Collection and Potential Risk Factors. Demo-} graphics, injury information, and treatment history were extracted from the medical records. We also contacted some patients to confirm details like occupation after fracture healing. All these data were used to evaluate the risk factors as follows:

Included demographics were age; gender; dominant arm (yes/no); hypertension (yes/no), defined as "systolic BP $\geq 140 \mathrm{mmHg}$, and/or diastolic BP $\geq 90 \mathrm{mmHg}$, and/or use of antihypertensive medicine within 2 weeks" [11]; diabetes mellitus (yes/no), defined as "a single raised glucose reading with symptoms or history of mellitus on medication or with the documented complications" [12]; former or current smokers who had smoked $>100$ cigarettes or reported regular cigar or cigarillo smoking were categorized as smoking (yes/no) [13]; alcohol abuse (yes/no), defined as alcoholic drinks $\geq 25$ g per day in the past 12 months [14]; and patients with $\mathrm{BMI} \geq 25$ were grouped as overweight (yes/no).

Injury information was fracture types of both radial head and ulna coronoid process. To measure the extent of medial instability, a valgus stress test was performed under fluoroscopy before surgery. Patients with joint space widening more than $3 \mathrm{~mm}$ were defined as MCL injury.

Surgical approach (lateral, posterior, and lateral with medial) and the time from initial injury to surgery were obtained through the treatment records. Occupations with a history of heavy use of upper extremities, which have been suggested in the previous study, were sorted into "heavy use" (yes/no), including manual laborer, weight lifter, farmers, health care, and child care [15].

2.3. Radiographic Assessment. Radiographic assessment was performed by two investigators (including one radiologist and one orthopedic surgeon). A consensus was made after a review by a senior surgeon if there were any disagreements. Preoperative radiographs were reviewed to identify the fracture types. Coronoid fractures were categorized according to the Regan and Morrey classification [16], and radial head fractures were classified based on the original Mason classification [17]. Since PTOA was reported more commonly in Mason III fractures [18], we divided radial head fractures type into groups I/II and III.

Postoperative radiographs were evaluated after surgeries and at the final follow-up after surgery. Subluxation was defined as incongruity of the ulnohumeral joint. PTOA was defined as the presence of radiographic change (joint space narrowing with/or osteophyte formation), and their severity was classified into four grades according to the Broberg and Morrey criteria [19]: grade 0 (without radiographic arthritis), grade 1 (slight joint space narrowing with/or minimum osteophyte formation), grade 2 (moderate joint space narrowing with/or moderate osteophyte formation), and grade 3 (severe degenerative change and joint destruction). All patients were sorted into the mild or no arthritis (grade 0 or 1 ) and moderate or severe arthritis (grade 2 or 3 ) groups.

2.4. Statistical Analyses. Group distributions were analyzed using an independent-sample $t$-test, and proportions among groups were compared by chi-square and Fisher's exact test. To identify potential predictors, we used univariate logistic analysis to access the association between demographics, injury information, treatment history, and the development of moderate or severe PTOA. The risk factors with a $p$ value $<0.05$ in the univariate analysis were selected for the multivariate logistic regression analysis. Hosmer-Lemeshow test was used to appraise the regression model fit to the data. Odds ratios (ORs) were presented with $95 \%$ confidence intervals (CIs), and a $p$ value $<0.05$ was set for significance. The incidence of moderate or severe PTOA was analyzed according to the number of risk factors with each patient. Finally, 
Mantel-Haenszel test was performed to analyze the correlation between injury types. Statistical analyses were performed using SPSS version 24.0.

\section{Results}

We excluded 92 cases among 290 consecutive TTI patients, because of an incomplete medical record for a minimum of 3 years $(n=29)$, a history of previous trauma or osteoarthritis $(n=15)$, and treatment with arthroplasty $(n=48)$. Finally, 198 patients with a mean follow-up of 42.8 months (range, 36-63 months) were analyzed in this study. Among them, $64(32.3 \%)$ patients had radiographic signs of moderate or severe PTOA with 60 classified as grade 2 and 4 as grade 3 . While in another group, 33 patients were grade 1 and 101 were without PTOA (grade 0). The demographics, injury, and treatment characteristics were shown in Table 1.

Univariate analysis indicated that six risk factors among 14 variables were potential predictors, including alcohol abuse, occupation, coronoid and radial head fracture types, MCL injury, and surgical approach (Table 2). An incidence greater than $50 \%$ was found in patients with type III coronoid fracture (68.2\%) and MCL injury (62.2\%). After multivariate logistic analysis, occupation with heavy use of upper extremities (OR 2.333, 95\% CI 1.060-5.136, $p=0.035$ ), Mason III radial head fracture (OR 4.049, 95\% CI 1.877-8.738, $p<$ 0.001), and MCL injury (OR 5.120, 95\% CI 1.261-20.790, $p=0.022$ ) were independently associated with the development of moderate or severe PTOA and constituted the final model (Table 3). The Hosmer-Lemeshow test indicated a good fit $(p=0.840)$ on $77.8 \%$ occasions. The incidences according to these three factors are shown in Figure 1. Furthermore, the incidence of moderate or severe PTOA increased substantially from a low incidence $(8.5 \%)$ in patients with no risk factors to a higher incidence $(58.6 \%)$ in the presence of two or more risk factors (Table 4). In addition, severe coronoid fracture was correlated with MCL injury $(R=0.663, p<0.001)$ and a more severe radial head fracture $(R=0.257, p<0.001)$.

All patients had a concentric reduction of ulnohumeral joint after surgery. However, subluxation was found in sixteen patients $(8.1 \%)$ within the first two weeks. Seven of them treated with a second surgery; the others were treated with external fixator or plaster. Besides, patients who suffered subluxation were more likely to develop moderate or severe PTOA $(p=0.007)$. Other complications included 17 cases of transient neuropathy (8.6\%) which disappeared within 4 months ( 2 weeks to 4 months), 11 cases of delayed union of the fractures (5.6\%), 4 cases of nonunion of the radial head that treated with resection, and 4 cases of local superficial infection (2\%) that healed after using antibiotics. Six cases had a hardware migration (3\%). The implant was removed in 4 of them, and the remaining 2 patients had a slight Kwire shift from the radial head without a secondary surgery. Forty-six patients had heterotopic ossification, of which 12 required a secondary surgery including heterotopic bone removal and elbow release. All these data were shown in Table 5.
TABLe 1: Patient demographics, injury, and treatment information.

\begin{tabular}{|c|c|c|c|}
\hline & $\begin{array}{c}\text { Mild or } \\
\text { no PTOA } \\
(n=134)\end{array}$ & $\begin{array}{l}\text { Moderate or } \\
\text { severe PTOA } \\
\quad(n=64)\end{array}$ & $p$ value \\
\hline Age, years, mean $( \pm S D)$ & $41.2( \pm 12.7)$ & $42.1( \pm 11.5)$ & 0.198 \\
\hline Gender, $n(\%)$ & & & 0.806 \\
\hline Male & $82(61.2)$ & $38(59.4)$ & \\
\hline Female & $52(38.8)$ & $26(40.6)$ & \\
\hline Dominance, $n(\%)$ & & & 0.822 \\
\hline Dominant & $71(53.0)$ & $35(54.7)$ & \\
\hline Nondominant & $63(47.0)$ & $29(45.3)$ & \\
\hline Diabetes mellitus, $n(\%)$ & $17(12.7)$ & $5(7.8)$ & 0.307 \\
\hline Alcohol abuse, $n(\%)$ & $45(33.6)$ & $31(48.4)$ & 0.044 \\
\hline Hypertension, $n$ (\%) & $35(26.1)$ & $12(18.8)$ & 0.254 \\
\hline Smoking, $n(\%)$ & $40(29.9)$ & $19(29.7)$ & 0.981 \\
\hline \multicolumn{4}{|l|}{ BMI } \\
\hline Mean $( \pm \mathrm{SD})$ & $23.6( \pm 2.8)$ & $24.4( \pm 2.9)$ & 0.383 \\
\hline Overweight, $n$ (\%) & $41(30.6)$ & $28(43.8)$ & 0.069 \\
\hline \multicolumn{4}{|l|}{ Occupation, $n(\%)$} \\
\hline Heavy use & $28(20.9)$ & $24(37.5)$ & 0.013 \\
\hline Coronoid fracture, $n(\%)$ & & & $<0.001$ \\
\hline $\mathrm{I}$ & $91(67.9)$ & $27(42.2)$ & \\
\hline II & $36(26.9)$ & $22(34.4)$ & \\
\hline III & $7(5.2)$ & $15(23.4)$ & \\
\hline Radial head fracture, $n(\%)$ & & & $<0.001$ \\
\hline $\mathrm{I}$ & $10(7.5)$ & $1(1.6)$ & \\
\hline II & $63(47)$ & $12(18.6)$ & \\
\hline III & $61(45.5)$ & $51(79.7)$ & \\
\hline MCL injury, $n(\%)$ & $17(12.7)$ & $28(43.8)$ & $<0.001$ \\
\hline $\begin{array}{l}\text { Time to surgery, } \\
\text { days, mean }( \pm S D)\end{array}$ & $4.9( \pm 3.5)$ & $5.5( \pm 4.4)$ & 0.311 \\
\hline Surgical approaches, $n(\%)$ & & & 0.018 \\
\hline Lateral & $95(70.9)$ & $34(53.1)$ & \\
\hline Posterior & $12(9.0)$ & $5(7.8)$ & \\
\hline Lateral with medial & $27(20.1)$ & $25(39.7)$ & \\
\hline
\end{tabular}

(\%) represents the percentage within each group.

\section{Discussion}

Moderate or severe elbow PTOA developed in $32.3 \%$ of patients on average 42.8 months after the terrible triad injury treated with ORIF. Occupation with heavy use of upper extremities, Mason III radial head fracture, and MCL injury significantly increased the risk of moderate or severe elbow PTOA as defined by the Broberg and Morrey grades 2-3. Besides, the presence of more risk factors was associated with a substantially increased incidence of moderate or severe elbow PTOA.

It is reported that Mason III radial head fracture is more likely to develop PTOA than Mason I or Mason II [18]. Complex radial fracture is difficult for surgical fixation. It increases the risk of poor reduction, which can lead to early PTOA [20]. However, some studies indicated that the 
TABLE 2: Univariate analysis: association between moderate or severe PTOA and patient demographics, injury, and treatment information.

\begin{tabular}{|c|c|c|c|c|c|}
\hline & Total $(n)$ & Moderate or severe PTOA & Odds ratio & $95 \%$ confidence interval & $p$ value \\
\hline Age & & & 1.006 & $0.982-1.031$ & 0.624 \\
\hline \multicolumn{6}{|l|}{ Gender (\%) } \\
\hline Male & 120 & $38(31.7)$ & 0.927 & $0.505-1.702$ & 0.806 \\
\hline Dominance (\%) & 106 & $35(33.0)$ & 0.934 & $0.514-1.698$ & 0.822 \\
\hline Diabetes mellitus (\%) & 22 & $5(22.7)$ & 0.583 & $0.205-1.659$ & 0.312 \\
\hline Alcohol abuse (\%) & 76 & $31(40.8)$ & 1.858 & $1.012-3.410$ & 0.046 \\
\hline Hypertension (\%) & 47 & $12(25.5)$ & 0.653 & $0.313-1.363$ & 0.256 \\
\hline Smoking (\%) & 59 & $19(32.2)$ & 0.992 & $0.517-1.903$ & 0.981 \\
\hline \multicolumn{6}{|l|}{ BMI (\%) } \\
\hline Overweight & 69 & $28(40.6)$ & 1.764 & $0.953-3.265$ & 0.071 \\
\hline \multicolumn{6}{|l|}{ Occupation (\%) } \\
\hline Heavy use & 52 & $24(46.2)$ & 2.271 & $1.179-4.375$ & 0.014 \\
\hline \multicolumn{6}{|l|}{ Coronoid fracture (\%) } \\
\hline I & 118 & $27(22.9)$ & Reference & Reference & Reference \\
\hline II & 58 & $22(37.9)$ & 2.060 & $1.041-4.076$ & 0.038 \\
\hline III & 22 & $15(68.2)$ & 7.222 & $2.671-19.528$ & $<0.001$ \\
\hline \multicolumn{6}{|l|}{ Radial head fracture (\%) } \\
\hline I/II & 86 & $13(15.1)$ & Reference & Reference & Reference \\
\hline III & 112 & $51(45.5)$ & 4.695 & $2.337-9.430$ & $<0.001$ \\
\hline MCL injury (\%) & 45 & $28(62.2)$ & 5.353 & $2.634-10.877$ & $<0.001$ \\
\hline Time to surgery & & & 1.045 & $0.968-1.127$ & 0.258 \\
\hline \multicolumn{6}{|l|}{ Surgical approaches (\%) } \\
\hline Lateral & 129 & $34(26.4)$ & Reference & Reference & Reference \\
\hline Posterior & 17 & $5(29.4)$ & 1.164 & $0.382-3.548$ & 0.789 \\
\hline Lateral with medial & 52 & $25(48.1)$ & 2.587 & $1.323-5058$ & 0.005 \\
\hline
\end{tabular}

(\%) represents the percentage of the total.

TABLE 3: Multivariate analysis: risk factors for moderate or severe PTOA.

\begin{tabular}{lccc}
\hline & Odds ratio & $\begin{array}{c}95 \% \text { confidence } \\
\text { interval }\end{array}$ & $p$ value \\
\hline Alcohol abuse & 1.445 & $0.716-2.914$ & 0.304 \\
Occupation (heavy use) & 2.333 & $1.060-5.136$ & 0.035 \\
Coronoid fracture & & & \\
$\quad$ I & Reference & Reference & Reference \\
II & 1.273 & $0.526-3.078$ & 0.592 \\
III & 3.616 & $0.798-16.376$ & 0.095 \\
Radial head fracture & & & \\
I/II & Reference & Reference & Reference \\
III & 4.049 & $1.877-8.736$ & $<0.001$ \\
MCL injury & 5.120 & $1.261-20.790$ & 0.022 \\
Surgical approaches & & & \\
$\quad$ Lateral & Reference & Reference & Reference \\
Posterior & 0.599 & $0.162-2.221$ & 0.443 \\
Lateral with medial & 0.319 & $0.070-1.460$ & 0.141 \\
\hline
\end{tabular}

displacement of the radial head was more related to the limitation of forearm rotation, not PTOA [21, 22]. Due to the different injury patterns between the TTI and isolated radial head fracture, it is still important to verify the relationship between PTOA and the severity of radial head fracture in the TTI. However, information is sparse. We found that moderate or severe PTOA tended to occur in the TTI patients with Mason III radial head fractures. It is known that increase stress on the articular surface can damage cartilage [20]. While compared with isolated radial head fractures, the TTI involves more structural damage of the elbow, including ligaments and coronoid process. Damage to these structures increases the valgus stress on the radial head [23, 24]. We also found that Mason III fractures were accompanied by more severe damage to the capsuloligamentous structures, reflecting higher energy damage to the elbow structures compared to Mason I or II fractures. Besides, in line with a previous study, we also found that Mason III fractures were more related to severe MCL injury [25]. It further increases the stress on the humeroradial joint which might be another reason.

It should be noted that we excluded patients treated with arthroplasty for radial fractures. Shore et al. [26] reported that $74 \%$ of patients developed PTOA after treating with metallic arthroplasty. And silicone implant was revealed to worsen arthritis secondary to elbow injury due to siliconitis [18]. We were unable to conduct a subgroup analysis for different treatments due to insufficient cases. It may not 


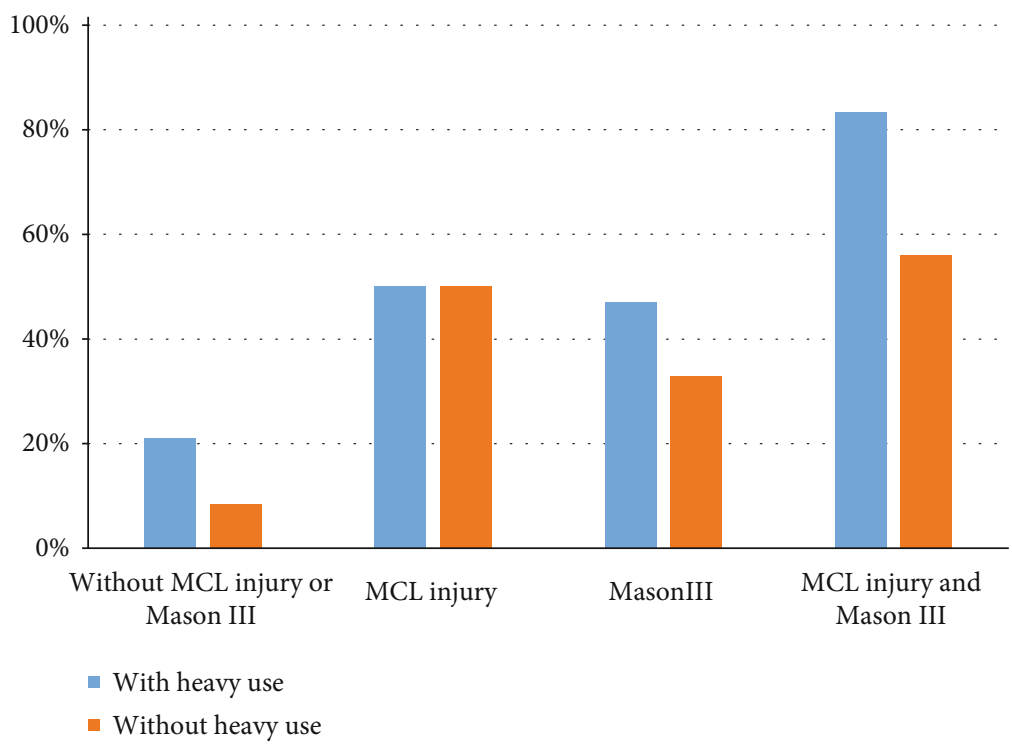

FIGURE 1: Incidence of moderate or severe PTOA according to presence or absence of heavy use of the elbow and an associated Mason III fracture and/or MCL injury.

TABLE 4: Incidence of moderate or severe PTOA according to the number of risk factors present.

\begin{tabular}{lcc}
\hline $\begin{array}{l}\text { Risk } \\
\text { factors }(n)\end{array}$ & $\begin{array}{c}\text { Patients }(n) \text { per risk } \\
\text { factor category }\end{array}$ & $\begin{array}{c}\text { Incidence of moderate or } \\
\text { severe PTOA }\end{array}$ \\
\hline 0 & 59 & $5(8.5 \%)^{\mathrm{a}}$ \\
1 & 81 & $25(30.1 \%)^{\mathrm{b}}$ \\
2 & 46 & $24(52.2 \%)^{\mathrm{c}}$ \\
3 & 12 & $10(83.3 \%)^{\mathrm{c}}$ \\
\hline
\end{tabular}

Statistical significance $p<0.05$ ( ${ }^{\mathrm{a}}$ compared to 1,2 , and 3 risk factors; ${ }^{\mathrm{b}}$ compared to 0,2 , and 3 risk factors; ${ }^{\mathrm{c}}$ compared to 0 and 1 risk factors).

TABLE 5: Complications between the two groups.

\begin{tabular}{lccc}
\hline Complications & $\begin{array}{c}\text { Mild or no PTOA } \\
(n=134)\end{array}$ & $\begin{array}{c}\text { Moderate or severe } \\
\text { PTOA }(n=64)\end{array}$ & $p$ value \\
\hline Heterotopic & 31 & 15 & 0.962 \\
ossification & 6 & 10 & 0.007 \\
Subluxation & 13 & 4 & 0.417 \\
Neuropathy & 8 & 3 & 0.712 \\
Delayed union & 2 & 2 & 0.445 \\
Nonunion & 5 & 1 & 0.405 \\
Hardware & 3 & 1 & 0.752 \\
migration & & &
\end{tabular}

influence our results because arthroplasty was commonly used to treat comminuted radial head fractures in the TTI. And in a series of 39 patients with TTI, Watters et al. [27] reported that PTOA was more common in patients with arthroplasty than ORIF ( $37 \%$ vs. $0 \%$ ).

In the current study, only $22.7 \%(45 / 198)$ of patients were diagnosed with "MCL injury," whereas Zhang et al. [28] reported an incidence of $71.4 \%$ (15/21) of MCL injury evalu- ated by magnetic resonance imaging (MRI). This difference is probably due to the various criteria. MRI can accurately detect partial tear of MCL, which is reported to heal in a way that does not affect prognosis after simple dislocations or fracture-dislocations of the elbow [29, 30]. In the report of Zhang et al., MCL injury was partially torn in 12 patients. And no one had a moderate or severe PTOA. We defined MCL injury as more than $3 \mathrm{~mm}$ of joint space widening by intraoperative fluoroscopy. It is used to find complete or large partial tears of MCL that can lead to instability of the medial side $[31,32]$. Our result was in line with another study, in which Jung et al. [33] showed that only $17.1 \%$ of TTI patients were defined as MCL injury with a criterion of more than $5 \mathrm{~mm}$ of joint space widening, which denoted a complete rupture of MCL. Although MCL is a primary stabilizer for the elbow, little is known about the relationship between MCL injury and PTOA. Our results are in accordance with a previous study which indicated a high incidence of PTOA in the presence of an associated MCL injury [34].

All TTI leads to different degrees of instability resulting from the extent of the separate component injury of the elbow [35]. It has been reported that the complete rupture of MCL is related to recurrent instability of the elbow increasing the risk of PTOA [33]. We also found that patients with subluxation of the elbow were more likely to develop moderate or severe PTOA. Recurrent instability of the elbow can damage the articular cartilage. On the other hand, it results in the lesion of the repaired collateral ligaments which further increased the instability. However, the necessity of repairing MCL is still under debate. In a series of 16 TTI patients, Toros et al. [36] indicated no differences in functional score and incidence of PTOA between patients with and without surgical treatment for MCL. In contrast, Jeong et al. [37] recommended MCL repair and reported no moderate or severe PTOA in their study of 13 TTI patients. An MCL injury is often accompanied by avulsion of the muscles 
from the epicondyle resulting in increased instability [38]. Moreover, the repair of MCL is an effective procedure if a postoperative instability occurs. Hence, the repair of severe MCL injury seems to have a positive effect on the prevention of PTOA. But, it needs to be confirmed by further prospective studies.

Occupation is known to be a risk factor for the development of osteoarthritis on several joints, such as the hip, knee, ankle, and elbow [15, 39-41]. However, Lübbeke et al. [42] reported that heavy work did not increase the PTOA of the ankle. It is not surprising because the risk factors for primary osteoarthritis and PTOA may differ due to the different pathogenesis of them. For example, occupation-related osteoarthritis commonly occurs within the ulnohumeral joint, while PTOA shows specific forms resulting from different injury patterns [43]. Also, in a series of 139 patients with surgical treatment of different elbow injuries, Guitton et al. [44] indicated that injury type, but not heavy use of the elbow, was associated with PTOA. Interestingly, we found heavy use of elbow was also a significant risk factor for PTOA in the patients of the TTI. A possible explanation is that cartilage health is depended on the interaction of biological, mechanical, and structural components [45]. Compensation from biological and mechanical components may keep the joint in a state of asymptomatic prearthritis after structural damage of the elbow [45], whereas the TTI is more complex among all elbow injuries and is often caused by high energy injury, which can destroy the homeostasis of both biological and structural components. Moreover, the reaction force of the elbow is more than twenty times as the external load because of the short lever arm of the muscles [46]. Then, under these conditions, cartilage degradation may be aggravated by mechanical stimulation during heavy use of the elbow.

Identifying the risk factors for PTOA and quantifying their effects are important for both doctors and patients. It allows for the establishment of prognosis, which could be used to inform patients of the future risk of PTOA. Besides, viable treatment options can be adopted to prevent the undesired outcome because some risk factors might be modifiable or their impact reduced. In our study, the only potentially modifiable risk factor was occupation after fracture healing. Moreover, we also assessed the risk categories according to the number of risk factors. And our results showed that the incidence of moderate or severe PTOA was lower in patients without heavy use of the elbow than those with (Figure 1). It indicated that patients should be informed of this additional risk and counseled about avoiding heavy use of the elbow (such as changing another type of job or switching the hand during these works), especially for those with Mason III radial fractures.

With regard to the influence of other potential factors, several studies found a correlation between age [41], gender [41], overweight [47, 48], smoking [13, 49], alcohol consumption [13], diabetes mellitus [48], hypertension [48], and primary osteoarthritis. Contrarily, they did not influence the development of moderate or severe PTOA in the current study. It further confirmed the difference in risk factors between primary osteoarthritis and PTOA, whereas we found that alcohol abuse was significant in univariable regression analyses, as well as the type of coronoid fracture and surgical approach. None of them were significantly associated with the development of moderate or severe PTOA in multivariable logistic regression analyses. The lack of significance may be due to interaction among variables. For example, we found that alcohol abuse mostly occurred in patients who were heavy users of the elbow, combined approach denoting severe damage to medial structures. Besides, a displaced coronoid fracture often represents an avulsion of the anterior MCL [38]. We also found that severe coronoid fracture had a correlation with MCL injury and a more severe radial head fracture.

\section{Limitations}

Several limitations of this report should be explained. First, being a retrospective study, there were unavoidable losses of follow-up and ambiguous medical records. We had contacted some patients to confirm details, but recall bias influenced the accuracy of those data. Second, due to its relatively low incidence of the TTI, most single-center studies of the risk factors for complications had a small number of patients. It decreased statistical power. Therefore, we conducted a multicenter study. Additional cases also allowed us to analyze more potential factors. However, choices of fixation (such as implants for fractures and treatments of MCL injuries) were depended on surgeons' discretion. It may interfere with our results even though surgeries were performed based on standard strategy. Third, we only evaluated PTOA by radiographs alone. Limited relationships were reported among radiographic evidence of PTOA and symptoms or disability [50]. Moreover, some authors would prefer a longer-term follow-up to analyze PTOA. But cartilage degeneration related to age should be considered under this condition. It has been suggested that PTOA develops 2 to 6 years after initial injuries [22]. Therefore, our mean followup of 42.8 months should be adequate to capture differences in the early development of PTOA.

\section{Conclusion}

Moderate or severe PTO was common (32.3\%) in TTI patients with surgical treatment. Risk increased with Mason III radial head fracture, MCL injury (medial instability), and heavy use of the elbow. The probability of developing moderate or severe PTOA is higher among patients with more risk factors. Patients should be counseled about avoiding heavy use of the elbow, especially for those with Mason III radial head fractures. In addition, surgeons should be aware of the recurrent instability. Further prospective studies are needed to confirm the effect of MCL treatment on the development of PTOA.

\section{Data Availability}

The clinical data used to support the findings of this study are restricted by the Ethics Committee of our hospitals in order to protect patient privacy. Data are available from the 
corresponding author for researchers who meet the criteria for access to confidential data.

\section{Conflicts of Interest}

The authors declare that they have no conflicts of interest.

\section{Authors' Contributions}

Weijun Guo made the conception and design. Di Lu, Qinglong $\mathrm{Li}$, Jing $\mathrm{Hu}$, and Ding $\mathrm{Xu}$ reviewed medical records and collected data from each institution. Then, Di Lu and Wenxiao Lin categorized groups. A consensus was made by Youming Zhao. Jingjing Li analyzed and interpreted those data independently. This article was drafted by Jinging Li then revised by Weijun Guo. All authors gave final approval for the version to be submitted.

\section{Acknowledgments}

We would like to acknowledge the departments from each institution for their support, as well as every participant for making this study possible. This study was supported by Wenzhou Science and Technology Bureau Project (Y20190270) and financially supported by the corresponding author Weijun Guo.

\section{References}

[1] D. Zhang, M. Tarabochia, S. Janssen, D. Ring, and N. Chen, "Risk of subluxation or dislocation after operative treatment of terrible triad injuries," Journal of Orthopaedic Trauma, vol. 30, no. 12, pp. 660-663, 2016.

[2] J. Zhang, M. Tan, and E. B. K. Kwek, "Outcomes of coronoidfirst repair in terrible triad injuries of the elbow," Archives of Orthopaedic and Trauma Surgery, vol. 137, no. 9, pp. 12391245, 2017.

[3] G. Giannicola, P. Calella, A. Piccioli, M. Scacchi, and S. Gumina, "Terrible triad of the elbow: is it still a troublesome injury?,” Injury, vol. 46, pp. S68-S76, 2015.

[4] H. Chen, G. Liu, and L. Wu, "Complications of treating terrible triad injury of the elbow: a systematic review," PLoS One, vol. 9, no. 5, article e97476, 2014.

[5] J. Rodriguez-Martin, J. Pretell-Mazzini, E. M. Andres-Esteban, and R. Larrainzar-Garijo, "Outcomes after terrible triads of the elbow treated with the current surgical protocols. A review," International Orthopaedics, vol. 35, no. 6, pp. 851-860, 2011.

[6] A. C. Gelber, M. C. Hochberg, L. A. Mead, N. Y. Wang, F. M. Wigley, and M. J. Klag, "Body mass index in young men and the risk of subsequent knee and hip osteoarthritis1," The American Journal of Medicine, vol. 107, no. 6, pp. 542-548, 1999.

[7] G. Mouzopoulos, N. K. Kanakaris, M. Mokawem, G. Kontakis, and P. V. Giannoudis, "The management of post-traumatic osteoarthritis," Minerva Medica, vol. 102, no. 1, pp. 41-58, 2011.

[8] B. W. Sears, G. J. Puskas, M. E. Morrey, J. Sanchez-Sotelo, and B. F. Morrey, "Posttraumatic elbow arthritis in the young adult: evaluation and management," The Journal of the American Academy of Orthopaedic Surgeons, vol. 20, no. 11, pp. 704-714, 2012.
[9] A. Celli and B. F. Morrey, "Total elbow arthroplasty in patients forty years of age or less," The Journal of Bone and Joint Surgery. American Volume, vol. 91, no. 6, pp. 1414-1418, 2009.

[10] D. M. W. Pugh, L. M. Wild, E. H. Schemitsch, G. J. W. King, and M. D. McKee, "Standard surgical protocol to treat elbow dislocations with radial head and coronoid fractures," The Journal of Bone and Joint Surgery. American Volume, vol. 86, no. 6, pp. 1122-1130, 2004.

[11] Z. Wang, Z. Chen, L. Zhang et al., "Status of hypertension in China," Circulation, vol. 137, no. 22, pp. 2344-2356, 2018.

[12] C. Y. Wen, Y. Chen, H. L. Tang, C. H. Yan, W. W. Lu, and K. Y. Chiu, "Bone loss at subchondral plate in knee osteoarthritis patients with hypertension and type 2 diabetes mellitus," Osteoarthritis and Cartilage, vol. 21, no. 11, pp. 1716-1723, 2013.

[13] I. K. Haugen, K. Magnusson, A. Turkiewicz, and M. Englund, "The prevalence, incidence, and progression of hand osteoarthritis in relation to body mass index, smoking, and alcohol consumption," The Journal of Rheumatology, vol. 44, no. 9, pp. 1402-1409, 2017.

[14] Y. Yang, X. Yang, F. Zhai, and Y. Cheng, "Dietary guidelines for Chinese (2016)," Journal of the Academy of Nutrition and Dietetics., vol. 116, no. 9, p. A37, 2016.

[15] L. K. Papatheodorou, M. E. Baratz, and D. G. Sotereanos, "Elbow arthritis: current concepts," The Journal of Hand Surgery, vol. 38, no. 3, pp. 605-613, 2013.

[16] W. Regan and B. Morrey, "Fractures of the coronoid process of the ulna," The Journal of Bone and Joint Surgery. American Volume, vol. 71, no. 9, pp. 1348-1354, 1989.

[17] M. L. Mason, "Some observations on fractures of the head of the radius with a review of one hundred cases," The British Journal of Surgery, vol. 42, no. 172, pp. 123-132, 1954.

[18] M. Chammas, "Post-traumatic osteoarthritis of the elbow," Orthopaedics \& Traumatology, Surgery \& Research, vol. 100, no. 1, pp. S15-S24, 2014.

[19] M. A. Broberg and B. F. Morrey, "Results of delayed excision of the radial head after fracture," The Journal of Bone and Joint Surgery. American Volume, vol. 68, no. 5, pp. 669-674, 1986.

[20] S. Strafun, I. Levadnyi, V. Makarov, and J. Awrejcewicz, "Comparative biomechanical analysis of stress-strain state of the elbow joint after displaced radial head fractures," J Med Biol Eng., vol. 38, no. 4, pp. 618-624, 2018.

[21] A. R. Kachooei and D. Ring, "Evaluation of radiocapitellar arthritis in patients with a second radiograph at least 2 years after nonoperative treatment of an isolated radial head fracture," Arch Bone Jt Surg., vol. 5, no. 6, pp. 375-379, 2017.

[22] M. J. Furey, D. M. Sheps, N. J. White, and K. A. Hildebrand, “A retrospective cohort study of displaced segmental radial head fractures: is $2 \mathrm{~mm}$ of articular displacement an indication for surgery?," Journal of Shoulder and Elbow Surgery, vol. 22, no. 5, pp. 636-641, 2013.

[23] M. Lapner and G. J. King, "Radial head fractures," The Journal of Bone and Joint Surgery. American Volume, vol. 95, no. 12, pp. 1136-1143, 2013.

[24] L. E. Karbach and J. Elfar, "Elbow instability: anatomy, biomechanics, diagnostic maneuvers, and testing," The Journal of Hand Surgery, vol. 42, no. 2, pp. 118-126, 2017.

[25] I. H. Rhyou, K. C. Kim, K. W. Kim, J.-H. Lee, and S. Y. Kim, "Collateral ligament injury in the displaced radial head and neck fracture: correlation with fracture morphology and 
management strategy to the torn ulnar collateral ligament," Journal of Shoulder and Elbow Surgery, vol. 22, no. 2, pp. 261-267, 2013.

[26] B. J. Shore, J. B. Mozzon, J. C. MacDermid, K. J. Faber, and G. J. W. King, "Chronic posttraumatic elbow disorders treated with metallic radial head arthroplasty," The Journal of Bone and Joint Surgery. American Volume, vol. 90, no. 2, pp. 271280, 2008.

[27] T. S. Watters, G. E. Garrigues, D. Ring, and D. S. Ruch, "Fixation versus replacement of radial head in terrible triad: is there a difference in elbow stability and prognosis?," Clinical Orthopaedics and Related Research, vol. 472, no. 7, pp. 2128-2135, 2014.

[28] C. Zhang, B. Zhong, and C.-F. Luo, “Treatment strategy of terrible triad of the elbow: experience in Shanghai 6th People's Hospital," Injury, vol. 45, no. 6, pp. 942-948, 2014.

[29] C. Forthman, M. Henket, and D. C. Ring, "Elbow dislocation with intra-articular fracture: the results of operative treatment without repair of the medial collateral ligament," The Journal of Hand Surgery, vol. 32, no. 8, pp. 1200-1209, 2007.

[30] J. B. Jupiter and D. Ring, "Treatment of unreduced elbow dislocations with hinged external fixation," The Journal of Bone and Joint Surgery. American Volume, vol. 84, no. 9, pp. 1630-1635, 2002.

[31] J. R. Bruce, R. Hess, P. Joyner, and J. R. Andrews, "How much valgus instability can be expected with ulnar collateral ligament (UCL) injuries? A review of 273 baseball players with UCL injuries," Journal of Shoulder and Elbow Surgery, vol. 23, no. 10, pp. 1521-1526, 2014.

[32] B. J. Erickson and A. A. Romeo, "The ulnar collateral ligament injury: evaluation and treatment," The Journal of Bone and Joint Surgery. American Volume, vol. 99, no. 1, pp. 76-86, 2017.

[33] S.-W. Jung, D.-H. Kim, S.-H. Kang, Y.-J. Eho, S.-W. Yang, and G.-E. Lee, "Risk factors that influence subsequent recurrent instability in terrible triad injury of the elbow," Journal of Orthopaedic Trauma, vol. 33, no. 5, pp. 250-255, 2019.

[34] T. Hatta, S. Nobuta, T. Aizawa et al., "Comparative analysis of surgical options for medial collateral ligament repair in terrible triad injury of the elbow," Orthopedic Reviews, vol. 8, no. 3, 2016.

[35] J. Nestorson, P.-O. Josefsson, and L. Adolfsson, "A radial head prosthesis appears to be unnecessary in Mason-IV fracture dislocation," Acta Orthopaedica, vol. 88, no. 3, pp. 315-319, 2017.

[36] T. Toros, K. Ozaksar, T. S. Sügün, M. Kayalar, E. Bal, and S. Ada, "The effect of medial side repair in terrible triad injury of the elbow," Acta Orthopaedica et Traumatologica Turcica, vol. 46, no. 2, pp. 96-101, 2012.

[37] W.-K. Jeong, J.-K. Oh, J.-H. Hwang, S.-M. Hwang, and W.-S. Lee, "Results of terrible triads in the elbow: the advantage of primary restoration of medial structure," Journal of Orthopaedic Science, vol. 15, no. 5, pp. 612-619, 2010.

[38] P. O. Josefsson, C. F. Gentz, O. Johnell, and B. Wendeberg, "Surgical versus non-surgical treatment of ligamentous injuries following dislocation of the elbow joint. A prospective randomized study," The Journal of Bone \& Joint Surgery, vol. 69, no. 4, pp. 605-608, 1987.

[39] E. C. Harris and D. Coggon, "HIP osteoarthritis and work," Best Practice \& Research. Clinical Rheumatology, vol. 29, no. 3, pp. 462-482, 2015.
[40] S. O. Ewalefo, M. Dombrowski, T. Hirase et al., "Management of posttraumatic ankle arthritis: literature review," Current Reviews in Musculoskeletal Medicine, vol. 11, no. 4, pp. 546557, 2018.

[41] M. Doherty, "Risk factors for progression of knee osteoarthritis," The Lancet, vol. 358, no. 9284, pp. 775-776, 2001.

[42] A. Lübbeke, D. Salvo, R. Stern, P. Hoffmeyer, N. Holzer, and M. Assal, "Risk factors for post-traumatic osteoarthritis of the ankle: an eighteen year follow-up study," International Orthopaedics, vol. 36, no. 7, pp. 1403-1410, 2012.

[43] D. Biswas, R. W. Wysocki, and M. S. Cohen, "Primary and posttraumatic arthritis of the elbow," Art, vol. 2013, pp. 1-6, 2013.

[44] T. G. Guitton, D. Zurakowski, N. C. van Dijk, and D. Ring, "Incidence and risk factors for the development of radiographic arthrosis after traumatic elbow injuries," The Journal of Hand Surgery, vol. 35, no. 12, pp. 1976-1980, 2010.

[45] T. P. Andriacchi, J. Favre, J. C. Erhart-Hledik, and C. R. Chu, "A systems view of risk factors for knee osteoarthritis reveals insights into the pathogenesis of the disease," Annals of Biomedical Engineering, vol. 43, no. 2, pp. 376-387, 2015.

[46] A. A. Amis, D. Dowson, and V. Wright, "Elbow joint force predictions for some strenuous isometric actions," Journal of Biomechanics, vol. 13, no. 9, pp. 765-775, 1980.

[47] J. Martel-Pelletier, A. J. Barr, F. M. Cicuttini et al., "Osteoarthritis," Nature Reviews. Disease Primers, vol. 2, no. 1, 2016.

[48] N. Yoshimura, S. Muraki, H. Oka et al., "Accumulation of metabolic risk factors such as overweight, hypertension, dyslipidaemia, and impaired glucose tolerance raises the risk of occurrence and progression of knee osteoarthritis: a 3-year follow-up of the ROAD study," Osteoarthritis and Cartilage, vol. 20, no. 11, pp. 1217-1226, 2012.

[49] A. K. Hedström, L. Klareskog, and L. Alfredsson, "Exposure to passive smoking and rheumatoid arthritis risk: results from the Swedish EIRA study," Annals of the Rheumatic Diseases, vol. 77, no. 7, pp. 970-972, 2018.

[50] A. L. C. Lindenhovius, K. M. Brouwer, J. N. Doornberg, D. C. Ring, and P. Kloen, "Long-term outcome of operatively treated fracture-dislocations of the olecranon," Journal of Orthopaedic Trauma, vol. 22, no. 5, pp. 325-331, 2008. 